\title{
CAPÍTULO XXXI
}

\section{LA FORMACIÓN CIENTÍFICA DISCIPLINAR DE ESTUDIANTES SORDOS EN EDUCACIÓN SUPERIOR, MEDIANTE EL USO DE LA TECNOLOGÍA DIGITAL}

\author{
José Rafael Proenza \\ PhD. Corporación Universitaria Iberoamericana. Correo electrónico: jose. \\ proenza@Ibero.edu.co.https://orcid.org/0000-0002-911-581
}

\author{
Carlos Enríquez Lozano \\ Magister. Corporación Universitaria Iberoamericana. Correo electrónico: carlos. \\ enriquez@ibero.edu.co.https://orcid.org/0000-0002-4938-8128
}

\author{
Sergio Serrano Galindo \\ Magister. Corporación Universitaria Iberoamericana. Correo electrónico: sergio. \\ serrano@ibero.edu.co.https://orcid.org/0000-0003-3525-4089
}

\section{Resumen}

El proyecto permitió superar determinadas barreras comunicativas y de acceso a la información para estudiantes Sordos en el nivel de educación superior, que impiden el desarrollo y apropiación de competencias en el quehacer científico. Por lo tanto, el objetivo general del proyecto está dirigido a desarrollar competencias en el campo de la investigación, para estudiantes Sordos universitarios, a partir del apoyo de una herramienta tecnológica digital, en Lengua de Señas Colombiana, en adelante LSC. Para tal fin, se partió de un diagnóstico hecho en la comunidad Sorda que estudia en la Corporación Universitaria Iberoamericana, que permitió establecer una relación de los resultados obtenidos por estos estudiantes en todas las asignaturas que se articulan con la investigación científica, desde las perspectivas del conocimiento, el saber utilizar esos conocimientos y los intereses y motivaciones, en torno a esta disciplina. Los objetivos específicos que se cumplieron se refieren a: determinar en los estudiantes Sordos el nivel de conocimientos, su aplicación y resultados alcanzados, en el área de la Metodología de la Investigación Científica, y elaborar una herramienta tecnológica, desde una perspectiva constructivista, que soportara la apropiación de dichos conocimientos y generara motivación positiva, en los estudiantes, en torno a los contenidos de la investigación científica, en su primera lengua. La investigación siguió un enfoque cualitativo con un tipo de estudio descriptivo y los métodos y técnicas, para materializar el cómo de la investigación desarrollada, fueron; la observación no participante, los grupos focales, la entrevista, y el uso de las tecnologías, en este caso una página web 2.0. Los resultados obtenidos; fueron muy positivos en el desarrollo de competencias

www.doi.org/10.47212/tendencias2020vol.xii.32 
científicas en los estudiantes y de una herramienta tecnológica digital, que fomenta las mismas, mediante el uso de su lengua materna.

Palabras clave: investigación científica, lengua de señas, Sordos en educación superior, tecnología digital.

\title{
SCIENTIFIC TRAINING DISCIPLINE OF DEAF STUDENTS IN HIGHER EDUCATION, THROUGH THE USE OF TECHNOLOGICAL TOOLS
}

\begin{abstract}
The project starts from the need to overcome communication barriers and access to information in the educational system for Deaf students at the higher education level, and that prevent the development and appropriation of competences in the scientific work. Therefore, the general objective of the project is aimed at contributing to the development of competences in the field of research, for Deaf university students, from the support and support of a digital technological tool, in Colombian Sign Language, from now on LSC. For this purpose, it was based on a diagnosis made in the Deaf community that studies at the Corporación Universitaria Iberoamericana, which allowed establishing a relationship of the results obtained by these students in all the subjects that are articulated with scientific research, from the perspectives of knowledge, knowing how to use that knowledge and the interests and motivations surrounding this discipline. The specific objectives that were fulfilled for the materialization of the project were: to determine in deaf students the level of knowledge, its application and results achieved, in the area of Scientific Research Methodology, and to develop a technological tool, from a constructivist perspective , that supports the appropriation of said knowledge and generates motivation in the students, around the disciplinary contents of scientific research in their first language. The methods and techniques, to materialize the how of the developed research, were; non-participant observation, focus groups, interviews, and the use of technologies, in this case a web 2.0 page. The results obtained; characterization of the level of development reached by Deaf students, around the competences for scientific research and the development of a technological tool supported in an essential way in LSC, which creates an effective learning environment in these students, for the development of their competences around scientific research.
\end{abstract}

Keywords: Deaf people in higher education, digital technology, scientific investigation, xsign language.

\section{Proyecto de investigación}

SEP INVESTIGA. Herramienta tecnológica para el aprendizaje de la metodología de la investigación científica, en estudiantes Sordos universitarios. 


\section{Entidades patrocinadoras}

Corporación Universitaria Iberoamericana de Colombia y Fondo de Desarrollo de la Educación Superior - FODESEP-, Colombia.

\section{Introducción}

La población Sorda en América Latina, ha logrado en los últimos años un considerable reconocimiento de sus derechos, con ello una mayor apertura para el acceso a la educación. Igualmente, en las Instituciones de educación, a partir del modelo de la educación inclusiva, se han creado ambientes más incluyentes, asequibles, de reconocimiento a la diversidad, en camino al logro de estándares de calidad para todos. No obstante, pese a los esfuerzos de las instituciones escolares, es innegable, que, aún existen muchas barreras para el pleno acceso a una educación de calidad para la población Sorda, mucho más aún, en los niveles de formación profesional, a la que acceden un número reducido de estas personas, y entre ellos, pocos logran graduarse. Uno de los procesos que reiteradamente reporta falta de accesibilidad para los estudiantes Sordos universitarios, es la investigación científica.

En la Corporación Universitaria Iberoamericana, a partir de su modelo pedagógico, y de la política establecida en torno a la educación inclusiva, la atención a la comunidad de estudiantes Sordos, es priorizada, razón por la cual se desarrollan investigaciones científicas, para optimizar este proceso. A partir de esta posición y considerando la lógica constructivista del aprendizaje, se ha procedido a tratar de encontrar la solución a dicho problema, mediante la participación activa de los propios estudiantes Sordos, considerando sus ideas, iniciativas y creatividad al respecto.

La investigación desarrollada, partió de plantear y buscar solución al siguiente problema; ¿Cómo lograr una comprensión eficiente y eficaz, de los contenidos de la investigación científica, en los estudiantes Sordos, que se forman como profesionales, en la Corporación Universitaria Iberoamericana, mediante el uso de la tecnología digital? Para el abordaje de esta pregunta problema, fue necesario establecer un objetivo general, el cual se definió en desarrollar competencias en el quehacer científico para los estudiantes Sordos universitarios, a partir del apoyo de una herramienta tecnológica digital, en Lengua de Señas Colombiana. 
Para ello, se partió de un diagnóstico realizado a la muestra seleccionada, (23 estudiantes sordos de diferentes programas profesionales) donde se identificaron el conjunto de conceptos y categorías que resultaban barreras comunicativas en el proceso de comprensión de la asignatura Metodología de la Investigación Científica, como base para el desarrollo de las competencias en el quehacer científico; y se procedió a la eliminación de esas barreras de comprensión, mediante su conceptualización en lengua de señas, además, se elaboró, con el uso de la tecnología digital, una herramienta para la comprensión académica de las principales categorías de la investigación científica, que da acceso, en LSC, a los contenidos fundamentales referidos a la Metodología de la Investigación Científica, por ser esta asignatura, común a todos los programas profesionales que se desarrollan en la universidad, y tener un contenido de difícil manejo por los estudiantes Sordos, reflejado en los resultados académicos analizados en el proceso de diagnóstico y los criterios emitidos en los grupos focales.

Es importante mencionar que en este estudio se aborda la categoría "Sordo" o "Sordos", con mayúscula inicial siempre, para referirse al enfoque socioantropológico de la sordera, donde se establece el reconocimiento de una comunidad de Personas con una Lengua y Cultura propias.

\section{Fundamentación teórica de la investigación}

Las sociedades actuales y su desarrollo están caracterizadas por la diversidad de culturas, contextos y sujetos que se articulan en un entramado social, político y económico, que hacen que las personas compartan una serie de características que las asemejan y otras que las hacen únicas. Lograr que todas estas diferencias confluyan en un mismo espacio y no sean los elementos que genere exclusión, es uno de los desafíos a los que se enfrenta la educación en la actualidad. Educar en este contexto presupone la presencia de tres indicadores fundamentales: calidad, equidad e inclusión; siendo la calidad y la equidad cruciales para garantizar la educación inclusiva y entendiendo, además, que la inclusión y la calidad deben ser variables recíprocas, en los modelos educativos de este siglo. (Fernández, 2017).

La educación inclusiva, es sinónimo de calidad, equidad, inclusión, reconocimiento y respeto a la diversidad, como condición humana que todos tenemos, sin temor a la 
censura, discriminación, segregación, señalamiento y en general exclusión. Es por ello, que la Educación Inclusiva es resaltada por organismos, instituciones y gobiernos de todo el mundo, ya que de ella depende el cumplimiento de objetivos importantes desde una perspectiva social que propende por el bienestar de todos, sobre la base del respeto a los derechos humanos y a la diversidad. Entre estas instituciones encontramos el Ministerio de Educación Nacional de Colombia (MEN), el cual resalta la importancia de llevar adelante en todo el país, una educación superior de calidad e incluyente, para formar profesionales capaces de desarrollar valores humanos y sociales; al respecto plantea: "La educación inclusiva está relacionada con la capacidad de potenciar y valorar la diversidad (entendiendo, protegiendo las particularidades), promover el respeto a ser diferente y garantizar la participación de la comunidad dentro de una estructura intercultural en los procesos educativos" (2013. p.4). Por otra parte, sobre la educación inclusiva, la Organización de las Naciones Unidas para la Educación, la Ciencia y la Cultura, (UNESCO, 2009) refiere, que la inclusión es un proceso que contribuye al reconocimiento de la diversidad y necesidades de todos, mediante una incorporación al aprendizaje y a todas las actividades dentro de la comunidad, para reducir exclusiones en los procesos educativos y tratar de acabar con las condiciones que permitan o favorezcan la exclusión.

Según los informes estadísticos de la Organización del Comercio y Desarrollo Económico, (OCDE), (citado por Rivas (2015) el acceso de las poblaciones más vulnerables a la educación en Colombia, ha sido mayor que en el siglo pasado, gracias a la creación de políticas favorables, que tributan a la eliminación de las principales barreras, que impedía este acceso, aunque aún dista mucho, lograr un óptimo de condiciones para estos estudiantes. El hecho de haber logrado mayor accesibilidad trae como consecuencia, que en las aulas confluya una gama de conocimientos y experiencias diferentes, pues muchos estudiantes provienen de diferentes culturas, hay formas y modos de vida distintos, que hacen que las situaciones educativas se tornen complejas, por lo que cada día, es más necesario que instituciones educativas y docentes, sean más competentes y comprometidos con la educación inclusiva. (Chiroleu, 2009).

La inclusión educativa en los estudiantes Sordos, igualmente resulta muy compleja, a decir de Sevilla et al., (2017), por la falta de preparación de los profesores, lo que ha 
motivado que la respuesta a la diversidad se desarrolle a través de modelos organizativos racionalistas, que pugnan por espacios y tiempos específicos de Educación Especial o de Educación Compensatoria para los alumnos "diversos". Los Sordos son una minoría lingüística y cultural, con una lengua propia, la lengua de señas, caracterizada por ser visual y no auditiva, y que constituye la mejor vía de comunicación y acceso a la información por parte de los estudiantes Sordos, pues esta es su lengua natural. No obstante, a ello, en las instituciones escolares, especialmente en las universidades, no encuentran ambientes lingüísticos, ni personal capacitado, para desarrollar los procesos docentes educativos en esta lengua, lo que afecta la comprensión eficiente y eficaz, de los estudiantes sordos en educación superior, y tributa de igual manera, a abandonos pedagógicos y deserción escolar por parte de los mismos. (Proenza et al., 2020).

Las escuelas con una orientación inclusiva, constituyen una vía fundamental para hacer efectivo el derecho a la educación en igualdad de condiciones, para contribuir al desarrollo de sociedades más justas, para fomentar una cultura de respeto y valoración de las diferencias sociales, culturales e individuales, (Hernández y Blanco, 2014). En este sentido, la educación inclusiva reconoce y atiende las necesidades, particularidades y singularidades de cada estudiante y aprende de estas diferencias, es por ello, que la “educación inclusiva está basada en principios de equidad, cooperación y solidaridad y valora las diferencias como oportunidad de enriquecimiento de la sociedad" (Mellado et al., 2017, p.3). En la educación inclusiva "el foco de atención es la transformación de los sistemas educativos y de las escuelas para que sean capaces de atender la diversidad de aprendizaje" Universidad Nacional a Distancia, (UNAD. 2015, p.24).

\section{La tecnología al servicio de la educación inclusiva}

El sistema educativo, como consecuencia del nuevo orden cultural, institucional y global, introducido por la multiplicidad de canales de información y medios, así como por la creciente diversidad étnica, cultural y lingüística, debe adaptarse a este nuevo contexto, donde el uso de la tecnología y sus posibles beneficios para todos los alumnos, constituye, sin duda alguna, uno de los principios básicos de esta manera de formar y educar a los futuros ciudadanos en el marco de una educación inclusiva. Esto es aún más necesario, en el caso de los estudiantes que biológicamente presentan alguna discapacidad sensorial, ya que el uso de la tecnología, puede soportar y estimular con 
mayor objetividad, los analizadores de los que dispone el estudiante, y así, suplir el déficit del analizador con bajo o ningún funcionamiento, en el caso de los estudiantes Sordos, usar tecnologías, que estimulen el analizador visual, es determinante en su aprendizaje. (Proenza et al., 2020)

"Las Tecnologías de la Información y Comunicación permiten desarrollar ciertos puntos claves que nos permitirán contemplar al estudiante como coprotagonista de su aprendizaje: aumentando la motivación a la hora de despertar interés por aprender y comprender; permitiendo la inmediatez de transmisión y recepción de información y aportando una flexibilidad de ritmo y de tiempo de aprendizaje" (Sevillano y Rodríguez, 2013, p.76).

En el mundo entero, se ha puesto la tecnología, en función de elevar la calidad de vida de las personas sordas, y luchar contra la principal barrera, que ellos tienen para la inclusión social, educativa y los aprendizajes. Si bien es cierto que no todas las tecnologías desarrolladas para personas sordas han logrado satisfacer de manera plena sus necesidades de acuerdo al fin para el que fueron creadas, si ha abierto la puerta para mejorar dichos trabajos siguiendo el rigor de la investigación científica. Destacan entre los productos fundamentales obtenidos, los de la Universidad de East Anglia y la productora británica de software de animación para televisión televirtual, que desarrolló un intérprete virtual (software) de la lengua de señas. El software consiste en un personaje de animación, cuyo nombre es Guido, que realiza la traducción de la Lengua de Signos Británica (BSL).

Por otra parte, el ingeniero colombiano Jorge Enrique Leal Rodríguez, patrocinado por la fundación HETAH (Herramientas Tecnológicas para Ayuda Humanitaria) y la empresa Tecnocontrol Supervisa S.A., han desarrollado un asistente virtual en lenguas de señas en línea, de acceso libre, que permite a la persona oyente tener una noción de cómo configurar un mensaje en lengua de señas mediante un avatar.

Un desarrollo japonés, describe un dispositivo telefónico de lengua de señas que permite a una persona sorda que utiliza la lengua de señas, conversar con una persona oyente en un lugar lejano y que no conoce la lengua de signos. El dispositivo se coloca en el lado de la persona sorda, y reconoce los movimientos de la mano de la lengua de 
señas y los traduce al lenguaje japonés. La trama en palabras japonesas se convierte en voces sintetizadas y se transmiten a un videoteléfono en el lado de la persona oyente. Las voces de la videoconferencia son reconocidas, y traducidas a lengua de señas para generar animaciones que se muestran en la pantalla de un televisor en el lado de la persona Sorda. (Hernández, Pulido y Arias, 2015).

La consideración de las nuevas tecnologías, para "todos" los alumnos como medio de aprendizaje en el ámbito educativo; sirve de puente de enlace para la innovación y el cambio dentro del contexto escolar. El uso de la tecnología y sus posibles beneficios para todos los estudiantes constituye uno de los principios básicos en la manera de formar y educar a los futuros ciudadanos. La escuela como consecuencia del nuevo orden cultural, institucional y global introducido por la multiplicidad de canales de información y medios, así como por la creciente diversidad étnica, cultural y lingüística, debe hacer frente a ese modelo de escolarización, con una gestión educativa, donde se ponga en primer orden, el desarrollo tecnológico, en función del aprendizaje y la formación integral de los estudiantes, especialmente aquellos, con evidentes barreras para la accesibilidad, como es el caso de los estudiantes sordos. (Fernández y Velasco, 2003).

Una de las funciones principales de la incorporación de la tecnología a la educación, está en lograr la optimización del proceso docente educativo, y con ello, elevar la calidad en la formación integral de los estudiantes, facilitando las vías de acceso a la información para todos, y lograr suplir, desde esta perspectiva, las dificultades perentorias en el proceso formativo. Las Tecnologías de la Información y Comunicación (TIC), como parte del desarrollo incorporado al proceso docente educativo, permite la atención a la diversidad, ya que por medio de estas herramientas se puede facilitar el acceso y la educación de calidad para todos. Es innegable que aún las poblaciones con discapacidad continúan siendo las más vulnerables en los contextos educativos y que a pesar del esfuerzo por el cambio de discurso y prácticas hacia la educación inclusiva, es necesario consolidar propuestas que respondan a dichas necesidades latentes e incrementar los esfuerzos para conseguir suplirlas en su totalidad, camino en el cual el uso de las tecnologías, evidentemente, facilita este proceso. (Romero et al., 2018). 
La investigación desarrollada se enfocó en lograr el potenciamiento de las competencias para la investigación científica, en los estudiantes Sordos de educación superior, mediante la creación de una herramienta tecnológica digital, que facilite en ellos el acceso, en su lengua natural, a los principales contenidos de las diferentes asignaturas que tributan a la formación de las competencias en la investigación científica. Se considera que el producto tecnológico es pertinente para todos los estudiantes Sordos, de las diferentes universidades del país, independientemente a la carrera, que hayan seleccionado, lo que le da a la investigación desarrollada, un nivel de usuario y de cobertura nacional, e incluso internacional, para aquellos Sordos colombianos, que se forman en universidades en el exterior y son usuarios de la LSC.

Es por ello, que, para utilizar las tecnologías, y especialmente las TIC, es importante tener presente una serie de recomendaciones, entre las cuales se proponen las siguientes:

Considerar el nivel de expectativas tanto de los estudiantes como de los docentes. Este tema es muy importante de cara a evitar un sentimiento de frustración por no conseguir los resultados esperados, pues cuando los estudiantes, no alcanzan a usar de manera correcta las tecnologías, más que ayudar, perjudica el proceso de formación.

Analizar al sujeto que va usar esa tecnología y el entorno. Hay que centrarse en el alumno, en sus procesos comunicativos, su capacidad comprensiva, expresiva y comunicativa, así como en el lenguaje y desarrollo de su pensamiento. Sobre esta base, se deben analizar cuáles son las adecuaciones y funcionalidades que conviene instalar en un determinado artefacto tecnológico. En el caso concreto de los estudiantes Sordos, recordar que el analizador visual es el determinante para su aprendizaje y que la audición no representa absolutamente nada para ellos, en este proceso.

Determinar necesidades y objetivos. Una vez determinadas las necesidades, se debe delimitar unos objetivos que sirvan como base para diseñar el software y los componentes informáticos adecuados.

La formación integral de los estudiantes, requiere de un conocimiento sólido, actual y útil, que se logra a través del uso de la tecnología, pero también el aprender a usar 
la tecnología, no solamente proporciona autonomía al estudiante, sino que genera un aprendizaje significativo y los prepara para el futuro, ya que estamos en la era de la tecnología, y la educación no es otra cosa que preparar al hombre para la vida, pero prepararlo bien. (Fernández y Velasco, 2003).

\section{Metodología}

La investigación fue de tipo cualitativa, su diseño fue amplio, flexible, enriquecedor, lo que dio la oportunidad, a los investigadores, de incorporar elementos metodológicos nuevos que enriquecieron los resultados, ejemplo de ello fue la participación activa de uno de los investigadores como "modelo señante" e informante clave.

La investigación siguió un enfoque cualitativo, con un diseño de investigación acción participativa. Se siguió un tipo de estudio exploratorio descriptivo, donde las principales técnicas para el control y seguimiento del proceso fueron: las observaciones sistemáticas, las entrevistas grupales a estudiantes Sordos, y grupos focales.

\section{Muestra}

La muestra de la investigación, a la que se le aplicaron las técnicas descritas anteriormente, con el propósito de recolectar la información necesitada, estuvo formada por 23 estudiantes Sordos de los programas de Educación Infantil, Educación Especial, Psicología, Contaduría y Administración y Finanzas. Igualmente participaron de la investigación, aportando información muy importante, cinco (5) intérpretes de lengua de señas, que acompañan a estos estudiantes, como mediadores comunicativos, en las diferentes actividades docentes que se desarrollan. De la misma manera, aportaron información valiosa, los profesores que desarrollan la asignatura de Metodología de la Investigación Científica, en los diferentes programas, que fueron cuatro (4) en total.

\section{Técnicas e instrumentos}

La metodología utilizada para la obtención de los datos requirió la aplicación de técnicas grupales de recolección de información, las cuales permitieron obtener un alto grado de consenso en torno al contenido, las categorías y los conceptos fundamentales de la investigación científica, que resultaban de mayor necesidad y de más difícil 
comprensión, para que fuera llevado a la herramienta tecnológica, en LSC. Las técnicas utilizadas fueron: La observación, la entrevista grupal y los grupos focales, mientras que los instrumentos se concretaron en las guías para la observación, la entrevista grupal, y la guía de entrevista que se desarrolló durante la ejecución de los grupos focales, ya que estas técnicas son de vital importancia en la obtención de información

\section{Procedimientos para la ejecución de la investigación}

La ejecución de la investigación se desarrolló en cuatro etapas o fases. A continuación, se mencionan y se describen cada una de ellas.

Etapa I. Conformación de la muestra de participantes de los grupos focales de estudiantes Sordos. Esta etapa se caracterizó por contactar a los estudiantes Sordos que se forman como profesionales en la Corporación Universitaria Iberoamericana y también la presencia de intérpretes de LSC, como garante de la fidelidad de los planteamientos que se expresaran.

Etapa II. Planificación y conducción de las entrevistas y grupos focales con los estudiantes Sordos. Esta etapa implicó la organización de las sesiones de trabajo con ellos, la recopilación de la información para el diagnóstico y la filmación de las mismas. En estas sesiones se obtuvieron las listas preliminares de los planteamientos de los dos grupos.

Etapa III. Análisis preliminar de los planteamientos y sobre la base de ello, la elaboración del diseño de la herramienta tecnológica (Página WEB 2.0).

Etapa IV. Elaboración de la herramienta, con el contenido y las categorías señaladas por los estudiantes Sordos, en torno a la investigación científica. En la elaboración de la herramienta participaron traductores oyentes de LSC y traductores Sordos, así como profesionales Sordos con experiencia en la investigación científica.

\section{Análisis y discusión de los resultados}

El proceso desarrollado desde la lógica de la investigación científica y el aprendizaje constructivista, y siguiendo un diseño flexible, contextualizado y participativo, con 
el propósito de obtener una información científica, confiable y sobre todo, solucionar el problema de partida, junto al cumplimiento de los objetivos general y específicos, además de la aplicación de las técnicas e instrumentos seleccionados descritas y el análisis y procesamiento de la información obtenida, desde una perspectiva cualitativa, se llegarona los siguientes juicios:

Los estudiantes Sordos, que formaron parte de la muestra, expresaron en las entrevistas y los grupos focales desarrollados, que hay algunas asignaturas, que por su naturaleza abstracta y el poco nivel de desarrollo del lenguaje disciplinar que tiene la LSC, les resultada muy difícil comprender, ya que la interpretación, por parte de los intérpretes de lengua de señas, en ocasiones no alcanza tampoco, niveles óptimos de desempeño, para poder entender, procesar y comprender las categorías y conceptos de asignaturas de un nivel alto de abstracción, como son las asignaturas referidas a la investigación científica, entre otras.

Luego de haber identificado las principales categorías y contenidos de la investigación científica, se procedió mediante el grupo de Traductores Sordos, generar una adaptación de alto contenido académico en LSC, lo cual se hizo mediante un ejercicio muy riguroso de reflexión lingüística en lengua de señas y con apoyo de intérpretes de LSC (personas oyentes), y los profesores de la asignatura, quienes además tienen experiencia en la investigación científica. Este proceso de adaptación lingüística y cultural a la persona Sorda y a la LSC, se materializó a través de vídeos con una alta calidad de resolución (formato $4 \mathrm{~K}$ ), de modo que las imágenes quedaran con toda la nitidez requerida para este tipo de productos, y así poder editar el contenido, desde diferentes dimensiones y que la información visual, llegue a los estudiantes Sordos, con claridad y objetividad para la comprensión de todos.

Una vez terminado todo el proceso descrito anteriormente, el contenido fílmico se llevó a una herramienta tecnológica digital WEB 2.0. La misma funciona de la siguiente manera: El desarrollo de esta plantilla se basa en Worpress es un sistema de gestión de contenidos (Content Management System o CMS) enfocado a la creación de cualquier tipo de página web. Está desarrollado en lenguaje PHP para entornos que ejecuten MySQL y Apache. Este sistema de gestión de contenidos puede también instalarse en 
entornos que ejecuten PostgreSQL, MariaDB y Nginx. Los datos que se publican se almacenan en una bbdd que hace las labores de archivo. Igualmente separa el contenido y el diseño en HTML y CSS. Cuenta también con diseño web responsive o adaptativo es una técnica de diseño web que busca la correcta visualización de una misma página en distintos dispositivos. Desde ordenadores de escritorio a tablets y móviles usando código media-queries de CSS3. El desarrollo es de código abierto (open source), bajo licencia GPLv2 esto significa que los desarrolladores pueden realizar mejoras e incorporan diferentes elementos, buscando así adaptarse a las necesidades de los usuarios. Se utilizó WordPress ya que también permite disfrutar de un mejor posicionamiento en Google lo que facilita que el producto aparezca en los buscadores a partir del desarrollo SEO básico. Cuenta con un panel de administración accesible de forma segura con un email y una contraseña.

Posee un certificado SSL (Secure Sockets Layer) que protege los datos transferidos por http mediante el cifrado activado por un certificado SSL en un servidor. Los certificados SSL contienen una clave pública y otra privada. La clave pública se utiliza para cifrar la información y la privada para descifrarla. Cuando un navegador se dirige a un dominio seguro, se produce una "presentación SSL" que autentica al cliente y el servidor y establece un método de cifrado y una clave de sesión única. Entonces pueden comenzar una sesión segura que protege la privacidad e integridad del mensaje. HTTPS utiliza el puerto 443, en lugar del puerto HTTP 80 en sus interacciones. De esta manera toda la información desde y hasta el visitante y servidor viajan por el Internet de forma segura. Certificado suministrado por Conceptika.co.

\section{Descripción del desarrollo web}

Cinco Banners principales animados de cada página, interfaz administrable muy intuitiva para el usuario, desarrollo de interfaz interactiva en WordPress, diseño web html5 adaptativo para dispositivos móviles, chat Interactivo Facebook y Whatsapp dentro de la web, galería de imágenes tipo portafolio digital, vinculación de video a la plataforma YouTube.

Este resultado WEB 2.0, actualmente está alojado bajo el dominio web de la Corporación Universitaria Iberoamericana, y se puede acceder a él mediante el link: 
http://sepinvestiga.edu.co, con libre acceso a todas las personas que lo quieran visualizar, pero esencialmente a todos los Sordos colombianos, que se forman como profesionales en las diferentes universidades del país, como una herramienta que les contribuye a un aprendizaje más eficaz y significativo, sobre la disciplina de la metodología de la investigación científica y que les tributa de una manera muy útil y objetiva, en la formación y desarrollo de sus competencias para la investigación científica.

\section{Conclusiones}

El proceso de investigación desarrollado, permitió corroborar aspectos fundamentales y esenciales en la formación de profesionales Sordos, signantes de la lengua de señas colombianas, ya que se evidenciaron conclusiones muy importantes que a continuación se plantean:

La educación inclusiva en el escenario de la investigación, es garantía para la formación exitosa de los estudiantes Sordos universitarios, y sobre todo el uso de la lengua de señas colombianas, en el marco de esta educación.

El uso de la tecnología digital en el proceso de formación de profesionales Sordos, es una exigencia y una necesidad, sentando las bases para elevar la calidad en el proceso de enseñanza aprendizaje y garantizando un aprendizaje significativo, con mayor autonomía, independencia y calidad, por parte de este tipo de estudiante.

\section{Referencias bibliográficas}

Chiroleu A. (2009). Políticas públicas de inclusión en la educación superior. Los casos de Argentina y Brasil. Pro-Posições, Campinas, v. 20, n. 2

Fernández, J. (2017). Educación inclusiva. Construyendo caminos para avanzar. Revista digital de Investigación en Docencia Universitaria. Vol 11. No 1. Lima. Ene-jul.

Fernández, J. y Velasco. N. (2003). Educación inclusiva y nuevas tecnologías: una convivencia futura y un diálogo permanente Pixel-Bit. Revista de Medios y Educación, núm. 21, julio, pp. 55-63 Universidad de Sevilla. España 
Hernández, C. Pulido, J. y Arias, J. (2015). Las tecnologías de la información en el aprendizaje de la lengua de señas. Revista Salud Pública. Volumen 17. No 1. P. 61-73. Recuperado el 14 de mayo del 2020. https://revistas.unal.edu.co/ index.php/revsaludpublica/article/view/36935/62013.

Hernández, R. Fernández, C y Badtista. P. (2014). Metodología de la Investigación. 6ta edición. Retomado: http://observatorio.epacartagena.gov.co/wpcontent/ uploads/2017/08/metodologia-de-la-investigacion-sexta-edicion. compressed.pdf. 12/05/2020

Hernández. L y Blanco. R (2014). Avances y desafios de la educación inclusiva en Iberoamérica. www.oei.es/noticias/spip.php?article13613

Mellado, M., Chaucono, J., Catrinao, Hueche, M., Oñate, Aravena, A. (2017). Percepciones sobre la educación inclusiva del profesorado de una escuela con Programa de Integración Escolar. Revista Educación, [S.l.], p. 1-14, dic. 2016. ISSN 2215-2644: <http://revistas.ucr.ac.cr/index.php/educacion/ article/view/21597>. Fecha de acceso: 12 jun. 2017 doi: http://dx.doi. org/10.15517/revedu.v41i1.21597.

MEN. (2013). La política de la educación superior inclusiva. Bogotá. Colombia 30 septiembre.

Proenza. J., Enríquez. C y Serrano. S. (2020). Herramienta tecnológica para la comprensión disciplinar de la investigación científica. Tendencias en la investigación universitaria. Una visión desde Latinoamérica. Vol. X. P. 420-435. Colección Unión Global. Fondo editorial Universidad Servando Garcés, Universidad Politécnica Territorial de Falcón Alonso Gamero. Venezuela.

Rivas, A. (2015). América Latina después de PISA: Lecciones aprendidas de la educación en siete países (2000-2015). Fundación CIPPEC. 
Romero. S, Sandoval. A, González. I, y Lozano. A. (2018). Herramientas tecnológicas para la educación inclusiva. Revista Tecnología, Ciencia y Educación, ISSN 2444-250X, ISSN-e 2444-2887, №. 9, págs. 83-112.

Sevilla, D. Martín, M. y Jenaro, C. (2017). Percepciones sobre la educación inclusiva: la visión de quienes se forman para docentes. Revista Investigación Educativa. No 25 Xalapa. Jul-Dic.

Sevillano, M. y Rodríguez, R. (2013). Integración de tecnologías de la información y comunicación en educación infantil en Navarra. Píxel-Bit. Revista de Medios y Educación, 42, pp. 75-87. Recuperado el 12 de octubre del 2013 de http://acdc.sav.us.es/pixelbit/images/stories/p42/06.pdf

UNAD. (2015). Educación inclusiva en la educación superior en Colombia y en la Universidad nacional abierta y a Distancia. República de Colombia.

UNESCO. (2009). Directrices sobre politicas de inclusión en la educación. Francia. Ed2009/ws/31.cld 1705.9. http://unesco.org/educacion/inclusive 